\title{
Will novel imaging approaches predict oligometastases or early liver metastasis in patients with colorectal cancer?
}

\author{
Bang-Bin Chen ${ }^{1,2}$ \\ ${ }^{1}$ Department of Medical Imaging, National Taiwan University Hospital, Taipei; ${ }^{2}$ Department of Medical Imaging, College of Medicine, National \\ Taiwan University, Taipei \\ Correspondence to: Bang-Bin Chen. Department of Medical Imaging, College of Medicine, National Taiwan University, No 7, Chung-Shan South Rd, \\ Taipei. Email: bangbin@gmail.com. \\ Provenance and Peer Review: This article was commissioned by the editorial office, Hepatobiliary Surgery and Nutrition. The article did not undergo \\ external peer review. \\ Comment on: Hazhirkarzar B, Khoshpouri P, Shaghaghi M, et al. Current state of the art imaging approaches for colorectal liver metastasis. \\ Hepatobiliary Surg Nutr 2020;9:35-48.
}

Submitted Sep 15, 2019. Accepted for publication Oct 24, 2019.

doi: $10.21037 /$ hbsn.2019.10.31

View this article at: http://dx.doi.org/10.21037/hbsn.2019.10.31

Colorectal cancer (CRC) is the third most common cancer worldwide and the second in the number of deaths per year. Diagnostic imaging is a pivotal part of clinical management at initial diagnosis, response evaluation, and recurrence surveillance. The comprehensive review by Hazhirkarzar and colleagues in this issue of HepatoBiliary Surgery and Nutrition has provided evidence of current state-of-the-art imaging evaluation in colorectal liver metastasis (CRLM) patients.

First, for the accurate diagnosis of liver metastasis, multiple imaging modalities have been investigated. The reported sensitivity of contrast-enhanced (CE)-CT, diffusion-weighted imaging (DWI)-MRI, extracellular matrix CE-MRI, and PET/CT were up to $84.6 \%, 82 \%$, $85.7 \%$, and $74.1 \%$, respectively. Of note, CT has a limitation for detecting CRLMs smaller than $1 \mathrm{~cm}$, and FDG-PET/CT is limited in mucinous tumors that have not FDG uptake. Lately, the combination of functional and detailed anatomical information from FDG-PET/MRI offers better diagnostic accuracy for local $\mathrm{T}$ and $\mathrm{N}$ staging than CE-CT, PET/CT or MRI alone. Besides, whole-body FDG-PET/MRI may serve as a one-stop-shop solution in a clinical setting, due to the ability to detect both intrahepatic and extrahepatic metastases.

Second, different imaging modalities offer crucial information for treatment planning, including surgery, local ablation, transarterial chemoembolization, selective internal radiation therapy, or systemic treatment. Both CT and
MRI can provide tumor burden and liver volumetry for the assessment of functional liver remnant before surgery, along with biliary and vasculature anatomy of the liver. MRI with the hepatobiliary contrast agent (Gd-EOB-DTPA) adds diagnostic performance for small hepatic metastases and can detect additional lesions that were missed by CT. Moreover, FDG PET/CT identified extrahepatic metastatic lesions in $17 \%$ of cases in a study of 65 patients with CRC, leading to a change in surgical management. Thus, it is recommended by the Royal College of Physicians as a pre-surgical evaluation tool. A recent study also reported that PET/MR outperformed the standard of care imaging in the accuracy of oncologic staging, and changed clinical management in $37.5 \%$ of times (1).

Finally, the quantitative imaging biomarkers have shown better performance than traditional morphologic-based criteria for predicting treatment response, recurrence, and survival. Some novel imaging biomarkers showed a significant correlation with pathological findings. For example, CT texture analysis parameters may represent tumor heterogeneity and cellularity. Apparent diffusion coefficient (ADC) derived from DWI-MRI is related to cell density and necrosis. Standardized uptake value (SUV) from PDG-PET is associated with the metabolic activity and aggressiveness of the tumor. These non-invasive imaging biomarkers can be used to predict prognosis before treatment, evaluate early treatment response, and decide optimal follow-up intervals. The optimal goal is to improve 
overall survival and life quality in these patients.

Despite the development of new imaging modalities and treatment techniques, the proportion of patients cured after surgery for CRLM has not significantly improved over the last decades. Recently, the concept of oligometastases has influenced clinical practice, particularly in CRC. Several randomized clinical trials have shown a survival benefit with the use of ablative interventions in patients with CRLM undergoing surgical resection. Even in patients with unresectable metastases, prolonged survival is still possible using metastasis-directed therapies, such as thermal ablation or stereotactic radiotherapy (2). The continuous improvement of imaging techniques is likely to increase the detection of oligometastasis. Thus, there remains a question of whether oligometastasis represents specific intermediate tumor biology or an early stage of diffuse disease. Currently, accurate predictive markers for oligometastatic tumors are still lacking. Neither clinical characteristics nor biological biomarkers can prospectively define which patient would likely benefit from targeting the metastatic sites. To answer this question, a prospective clinical trial of metastasisdirected treatment using novel functional imaging biomarkers will be necessary.

Thus, developing strategies to prevent metastasis, or to detect it at its earliest stages, is a priority for improving patient outcomes. Recently, there is growing evidence that primary tumors precondition the microenvironments of the future metastatic site to form so-called "pre-metastatic niches". This concept of organotropism was first introduced by Stephen Paget in 1889 (3). Different types of cancers have different preferences for metastatic targets, suggesting that certain types of cancer are inclined to migrate to and flourish in specific microenvironments (4). This 'primed' microenvironment helps promote tumor cell homing, colonization, and ultimately growth at the target organ. Once metastases grow at niche sites, curative options became limited, and $90 \%$ of cancer-associated deaths are related to complications from metastasis.

Radiomic converts medical images such as CT into high-throughput quantitative data that can be used to predict CRLM. Using CT texture analysis, prior studies have identified either uniformity or entropy of the liver parenchyma as potential predictors for the development of CRLM. Rao et al showed that entropy values of the liver parenchyma at staging CT might differ between patients who eventually develop liver metastases and those who do not (5). Patients with synchronous metastatic disease had higher entropy and lower uniformity than those with no metastatic disease (5). Also, Beckers et al found that uniformity was a significant predictor for early metastases ( $\leq 6$ months), but none of the CT texture parameters could predict intermediate/late metastases (6). Even using segmental CT rather than whole-volume CT, they failed to demonstrate clear predictive value to identify patients at risk of developing metachronous metastases within 2 years (7). Based on these preliminary results, it seems that pre-metastatic niches may be detected using CT radiomics, especially for predicting early metastasis. Future work that combines radiomics, machine learning or deep learningbased approaches to predict CRLM will be a fascinating and clinically beneficial area of investigation.

In conclusion, the recent improvements in medical imaging techniques have greatly enhanced our assessment of liver metastasis and guided personalized treatment approaches. Further studies aiming at better defining early clinical and biological characteristics of these patients are needed to increase the number of cured patients with CRLM.

\section{Acknowledgments}

Funding: None.

\section{Footnote}

Conflicts of Interest: The author has completed the ICMJE uniform disclosure form (available at http://dx.doi. org/10.21037/hbsn.2019.10.31). The author has no conflicts of interest to declare.

Ethical Statement: The author is accountable for all aspects of the work in ensuring that questions related to the accuracy or integrity of any part of the work are appropriately investigated and resolved.

Open Access Statement: This is an Open Access article distributed in accordance with the Creative Commons Attribution-NonCommercial-NoDerivs 4.0 International License (CC BY-NC-ND 4.0), which permits the noncommercial replication and distribution of the article with the strict proviso that no changes or edits are made and the original work is properly cited (including links to both the formal publication through the relevant DOI and the license). See: https://creativecommons.org/licenses/by-nc$\mathrm{nd} / 4.0 /$. 


\section{References}

1. Amorim BJ Hong TS, Blaszkowsky LS, et al. Clinical impact of PET/MR in treated colorectal cancer patients. Eur J Nucl Med Mol Imaging 2019;46:2260-9.

2. Pitroda SP, Chmura SJ, Weichselbaum RR. Integration of radiotherapy and immunotherapy for treatment of oligometastases. Lancet Oncol 2019;20:e434-42.

3. Paget $\mathrm{S}$. The distribution of secondary growths in cancer of the breast. 1889. Cancer Metastasis Rev 1989;8:98-101.

4. Aguado BA, Bushnell GG, Rao SS, et al. Engineering the pre-metastatic niche. Nat Biomed Eng 2017. doi: 10.1038/ s41551-017-0077.

Cite this article as: Chen BB. Will novel imaging approaches predict oligometastases or early liver metastasis in patients with colorectal cancer? HepatoBiliary Surg Nutr 2020;9(3):391-393. doi: $10.21037 / h b s n .2019 .10 .31$
5. Rao SX, Lambregts DM, Schnerr RS, et al. Whole-liver CT texture analysis in colorectal cancer: Does the presence of liver metastases affect the texture of the remaining liver? United European Gastroenterol J 2014;2:530-8.

6. Beckers RCJ, Lambregts DMJ, Schnerr RS, et al. Whole liver CT texture analysis to predict the development of colorectal liver metastases-A multicentre study. Eur J Radiol 2017;92:64-71.

7. Beckers RCJ, Beets-Tan RGH, Schnerr RS, et al. Wholevolume vs. segmental CT texture analysis of the liver to assess metachronous colorectal liver metastases. Abdom Radiol (NY) 2017;42:2639-45. 